\title{
An Efficient Downlink Radio Resource Allocation with Carrier Aggregation in LTE-Advanced Networks
}

\author{
Hong-Sheng Liao, Po-Yu Chen, Member, IEEE, and Wen-Tsuen Chen, Fellow, IEEE
}

\begin{abstract}
In long term evolution-advanced (LTE-A) networks, the carrier aggregation technique is incorporated for user equipments (UEs) to simultaneously aggregate multiple component carriers (CCs) for achieving higher transmission rate. Many research works for LTE-A systems with carrier aggregation configuration have concentrated on the radio resource management problem for downlink transmission, including mainly CC assignment and packet scheduling. Most previous studies have not considered that the assigned CCs in each UE can be changed. Furthermore, they also have not considered the modulation and coding scheme constraint, as specified in LTE-A standards. Therefore, their proposed schemes may limit the radio resource usage and are not compatible with LTE-A systems. In this paper, we assume that the scheduler can reassign CCs to each UE at each transmission time interval and formulate the downlink radio resource scheduling problem under the modulation and coding scheme constraint, which is proved to be NP-hard. Then, a novel greedy-based scheme is proposed to maximize the system throughput while maintaining proportional fairness of radio resource allocation among all UEs. We show that this scheme can guarantee at least half of the performance of the optimal solution. Simulation results show that our proposed scheme outperforms the schemes in previous studies.
\end{abstract}

Index Terms-LTE-A networks, carrier aggregation, radio resource allocation

\section{INTRODUCTION}

$I^{N}$ $\mathrm{N}$ recent years, the number of users using intelligent hand-held equipments increases significantly due to rapid development of information and communication technologies and novel applications. There are tremendous demands of wireless services with high bandwidth, such as video streaming, video conference, and other services, offered by services vendors to make life more convenient. International Mobile Telecommunications-Advanced (IMTAdvanced), defined by International Telecommunication Union (ITU) for 4G systems, requires that mobile communication systems meet a requirement of a downlink peak data rate of $1 \mathrm{Gbps}$ and an uplink peak data rate of $500 \mathrm{Mbps}$ [1]. To achieve the peak data rate required by IMT-Advanced, Long Term Evolution-Advanced (LTE-A) under the 3rd Generation Partnership Project (3GPP) specifies that user equipments (UEs) support bandwidth up to $100 \mathrm{MHz}$. However, various slots of the spectrum have been assigned to existing legacy systems in many countries so that such a large contiguous frequency band is rarely available. To address this issue, carrier aggregation (CA) technique was introduced under LTE-A networks [2]. With CA technique,

- H.-S. Liao is with the Information Security and Privacy, Chunghwa Telecom Co. Ltd., Taipei, Taiwan. E-mail: hayate5398@hotmail.com.

- P.-Y. Chen is with the Institute of Information Science, Academia Sinica, Nankang, Taipei 115, Taiwan. E-mail: chenpy@mx.nthu.edu.tw.

- W.-T. Chen is with the Institute of Information Science, Academia Sinica, Nankang, Taipei 115, Taiwan and the Department of Computer Science, National Tsing Hua University, Hsinchu 300, Taiwan.

E-mail:chenwt@iis.sinica.edu.tw.

Manuscript received 6 Feb. 2013; revised 27 Nov. 2013; accepted 23 Dec. 2013, published online $x x x x x$.

For information on obtaining reprints of this article, please send e-mail to: tmc@computer.org, and reference IEEECS Log Number TMC-2013-02-0062.

Digital Object Identifier no. 10.1109/TMC.2013.2297310
UEs can simultaneously aggregate two or more different frequency fragments (e.g., $20 \mathrm{MHz}$ ), called component carriers (CCs), to form wider transmission bandwidth (e.g., $100 \mathrm{MHz}$ ) for achieving higher transmission rate. Since CA supports contiguous and non-contiguous CC aggregation, a UE can aggregate multiple CCs that are in the same or different bands to utilize frequency fragments efficiently [3]. CA is also designed to maintain backward compatibility for LTE Release 8/9 UEs [3].

A review of previous research works, which will be detailed in Section 2, indicates that many aspects of the functionality of CA are still needed to be investigated. One of them deals with radio resource management for downlink transmission, including CC assignment, packet scheduling, and other functions [2]. CC assignment determines which CCs are efficiently assigned to each UE. Packet scheduling is referred to the task of allocating time-frequency resources of CCs, called resource blocks (RBs), as well as modulation and coding schemes (MCSs) to UEs at each transmission time interval (TTI). The assignment of CCs to each UE can be reconfigured by radio resource control for the purpose of balancing the traffic load over multiple CCs or increasing the channel quality of UEs. However, most previous studies have not considered that the assigned CCs in each UE can be changed. It may limit the radio resource usage when the traffic loads or the channel conditions of CCs vary. Moreover, the MCS constraint, as specified in 3GPP TR 36.912 [10], requires that only one MCS can be selected for each assigned CC across all its assigned RBs for a UE at any TTI in absence of Multiple Input Multiple Output (MIMO) spatial multiplexing. The main reason of introducing this MCS constraint is due to implementation cost for UEs. Whenever a UE is required to support an 
additional MCS in a CC, it needs an extra transceiver to demodulate the mixed signal when there is only one antenna in UE, which is the case in most existing and upcoming devices in recent years. The extra transceiver will increase the hardware cost, the size of circuit, and power consumption. However, the MCS constraint has not been considered in all previous studies. They assume that the MCS for a UE can be selected independently for each RB. In other words, a UE can select two or more MCSs for different RBs of its assigned CC.

Motivated by the aforementioned considerations, we formulate the downlink radio resource scheduling problem for LTE-A systems with CA configuration under the following assumptions: (i) The scheduler can reassign CCs to each UE at each TTI; (ii) Only one MCS can be selected for each assigned CC across all its assigned RBs for a UE at any TTI. In this paper, we prove that the scheduling problem is NPhard under the backlogged traffic model, and subsequently we propose a novel greedy-based scheme to assign RBs of each CC and MCSs to UEs at each TTI for downlink transmission. The goal is to maximize the system throughput while maintaining proportional fairness of radio resource allocation among all UEs. It is noted that it is important to maintain proportional fairness among all UEs in order to prevent starvation of UEs, which may suffer from bad channel conditions when they are deployed in cell-edge or interfered by noises and other signals.

In the proposed scheme, we first formulate an assignment of a CC with its associated MCS to a UE as a gain and calculate gains of all combinations of UEs, CCs, and MCSs in each iteration. An assignment with higher gain indicates that the UE can transmit more data by using the MCS with higher rate on the assigned CC and has lower average transmission rate over the accumulated time. Then, iteratively the highest-gain assignment is chosen until all UEs are assigned CCs together with MCSs or no better assignment with higher gain can be found. We show that the proposed scheme can guarantee at least half of the performance of the optimal solution under the backlogged traffic model.

The rest of this paper is organized as follows. In Section 2, we review related works on the radio resource scheduling problem. In Section 3, we introduce the time-frequency resource structure of LTE/LTE-A, describe the downlink system model, formulate the radio resource scheduling problem, and prove that the problem is NP-hard. In Section 4 , we describe the proposed greedy-based scheme, and subsequently we analyse its computational complexity and show bound of its performance with the optimal solution. In Sections 5 and 6, we give the simulation results and conclude this paper, respectively.

\section{Related Works}

The downlink radio resource management problems for LTE-A systems with CA configuration have been widely studied [4], [5], [6], [7], [8], [9]. The schemes that they proposed for the problems are mainly based on the following procedures. They first use a load balancing scheme to assign CCs to UEs of LTE Release 8/9 and LTE-A. After CCs are assigned, they then schedule UEs at each TTI according to a packet scheduling scheme to optimize the radio resource usage of the system.

In [4], [5], the authors introduced the inter-band CA scenario, where UEs aggregate multiple CCs of different frequency bands with various radio propagation characteristics (e.g., coverage), and proposed load balancing schemes for the inter-band CA scenario to achieve higher performance. In [6], [8], [9], the authors modified the wellknown proportional fair scheduler [7] to perform joint scheduling in order to address the scheduling problem for optimizing the radio resource usage while maintaining proportional fairness among all UEs of LTE Release 8/9 and LTE-A. The proportional fair scheduler let the system achieve high throughput and maintain proportional fairness among all UEs by giving higher priority to UEs with higher current transmission rate and lower average transmission rate in the past. They proposed joint scheduling to improve the overall throughput performance by considering the historical average throughput of UEs from all CCs. They showed that the joint scheduling method can achieve higher system throughput than independent scheduling method performed on each CC. However, they did not considered that the assigned CCs in each UE can be changed dynamically according to channel quality and also the MCS constraint has not been considered in the above works.

The scheduling problem with the MCS constraint for LTE systems has been addressed by Kwan et al. [11]. They formulated an optimization problem in order to maximize the system throughput. They proposed a simple greedy-based scheme to reduce the computational complexity of the optimization problem and showed that the optimal scheme can achieve higher performance than the sub-optimal scheme at the expense of a higher complexity. The works in [12], [13] [14] followed the above optimization problem and proposed greedy-based or meta-heuristic schemes to get sub-optimal solutions. In [15], Kwan et al. proposed another optimization problem for either maximizing the system throughput or achieving proportional fairness among all UEs. However, the above works are designed for LTE systems without CA configuration, and the computational complexity of their proposed schemes has not been clearly stated.

\section{BACKGROUND AND FORMULATION}

\subsection{LTE/LTE-A Frame Structure}

LTE/LTE-A employs orthogonal frequency division multiple access (OFDMA) technique, which is based on fixed frame-based transmission, for downlink transmission. In OFDMA, a radio frame with $10 \mathrm{~ms}$ duration is divided into 10 equal-sized sub-frames each with $1 \mathrm{~ms}$ duration. Each sub-frame is composed of two equal-sized time-slots with $0.5 \mathrm{~ms}$ duration. Each slot corresponds to 7 or 6 consecutive OFDM symbols depending on the cyclic prefix length. A basic scheduling unit in LTE/LTE-A, called a resource block, consists of a time-slot in the time domain and 12 consecutive subcarriers in the frequency domain. Each subcarrier has a $15 \mathrm{kHz}$ bandwidth [2], [16]. Basic time-frequency resource structure of LTE/LTE-A (normal cyclic prefix case) is shown in Fig. 1. 


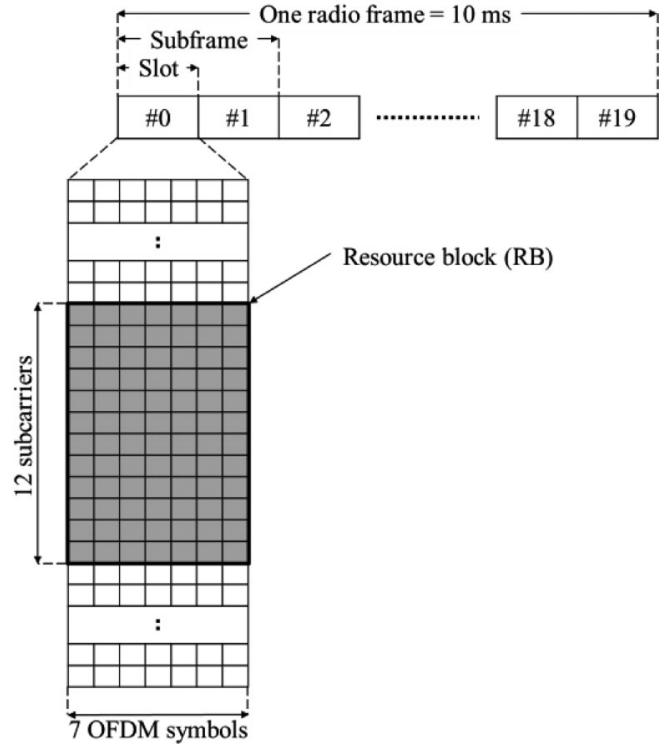

Fig. 1. Basic time-frequency resource structure of LTE/LTE-A (normal cyclic prefix case).

LTE downlink scheduling, referred as the task of allocating RBs of a CC to UEs, is performed at each TTI (i.e., subframe duration of $1 \mathrm{~ms}$ ). In contrast, LTE-A downlink scheduling is further enhanced to allocate RBs of multiple CCs to UEs.

\subsection{Downlink System Model}

In this paper, we consider a downlink scenario in LTE-A networks with an E-UTRAN NodeB (eNB) (i.e., the base station in LTE-A) and $m$ active UEs. The eNB can employ $n$ CCs to transmit data, and each UE can employ at most $z$ CCs to receive data. Each CC has the same bandwidth of $p$ RBs. Downlink scheduling is performed at each TTI. Multiple RBs of CCs can be allocated to a single UE at each TTI, while each RB of a CC can be assigned up to one UE (in absence of MIMO). In this paper, we assume the backlogged traffic model that the eNB always has data for transmission to every UE at each TTI. Thus the eNB can schedule all RBs of all CCs to UEs at each TTI.

The channel conditions may vary with time, frequency, and UE's location. We assume that each RB of each CC for a UE has time varying channel condition. Each UE can estimate individually the channel quality on each RB of each CC by using reference signals transmitted from the eNB. The feedback reports of the estimated channel quality are transmitted by each UE to the eNB in form of channel quality indicators (CQIs) [2]. A CQI value can be mapped to the highest-rate MCS that can be used by a UE for an RB [17]. We assume that the scheduler knows the channel conditions across all UEs and all RBs of all CCs from the CQIs reported by all UEs. Furthermore, there are $q$ available MCSs that can be used, where MCS 1 has the lowest transmission rate and MCS $q$ has the highest transmission rate. Let $r_{l}$ be the achieved transmission rate for a UE on an RB with MCS $l$. Let $Q_{i, j, k}$ be the index of the highest-rate MCS that can be used by UE $i$ on RB $k$ of CC $j$.

For convenience, we have listed the symbols used throughout the paper in Table 1.
TABLE 1

A List of Symbols Used in This Paper

\begin{tabular}{|c|l|}
\hline Symbol & \multicolumn{1}{c|}{ Definition } \\
\hline$i$ & UE index, $i \in \boldsymbol{M}:=\{1, \ldots, m\}$ \\
\hline$j$ & CC index, $j \in \boldsymbol{N}:=\{1, \ldots, n\}$ \\
\hline$k$ & RB index, $k \in \boldsymbol{P}:=\{1, \ldots, p\}$ \\
\hline$t$ & MCS index, $l \in \boldsymbol{Q}:=\{1, \ldots, q\}$ \\
\hline$r_{l}$ & TTI index \\
\hline$Q_{i, j, k}$ & $\begin{array}{l}\text { The achieved transmission rate for a UE } \\
\text { on an RB with MCS } l\end{array}$ \\
\hline$w_{i}$ & $\begin{array}{l}\text { The index of the highest-rate MCS that } \\
\text { can be used by UE } i \text { on RB } k \text { of CC } j\end{array}$ \\
\hline$x_{i, j, k, l}$ & $\begin{array}{l}\text { A priority weight of UE } i \\
\text { or not RB } k \text { of CC } j \text { with MCS } l \text { is as- } \\
\text { signed to UE } i\end{array}$ \\
\hline$r_{i, j, k, l}$ & $\begin{array}{l}\text { The achieved transmission rate for UE } i \\
\text { on RB } k \text { of CC } j \text { with MCS } l\end{array}$ \\
\hline $\boldsymbol{U}$ & $\begin{array}{l}\text { The set of all pair }(i, j) \text { of possible as- } \\
\text { signments of CC } j \text { to UE } i\end{array}$ \\
\hline$V(j, k)$ & $\begin{array}{l}\text { The weighted transmission rate of the } \\
\text { UE currently being assigned RB } k \text { of CC } \\
j\end{array}$ \\
\hline$v(i, j, k, l)$ & $\begin{array}{l}\text { The weighted transmission rate of UE } i \\
\text { on RB } k \text { of CC } j \text { with MCS } l\end{array}$ \\
\hline$g(i, j, l)$ & $\begin{array}{l}\text { The gain of the assignment of CC } j \text { with } \\
\text { MCS } l \text { to UE } i\end{array}$ \\
\hline$N_{i, j}$ & The set of RBs of CC $j$ assigned to UE $i$ \\
\hline
\end{tabular}

\subsection{Problem Formulation}

The objective of the well-known proportional fair scheduler is to maximize the utility function $\sum_{i} \log \mu_{i}(t)$ (known as proportional fair criteria) in order to achieve high throughput while maintaining proportional fairness of radio resource allocation among all users [18], [19], where $\mu_{i}(t)$ is the average transmission rate of user $i$ up to TTI $t$. In [18], [19], it has been shown that the scheduler can maximize the utility function $\sum_{i} \log \mu_{i}(t)$ by maximizing the objective function $\sum_{i} x_{i}(t) R_{i}(t) / \mu_{i}(t)$, where $x_{i}(t)$ is an indicator variable to denote whether or not user $i$ is scheduled at TTI $t$, and $R_{i}(t)$ is the current transmission rate of user $i$ at TTI $t$. In the above objective function, $1 / \mu_{i}(t)$ can be regarded as a priority weight $w_{i}(t)$ of user $i$ at TTI $t$ in the sense that the user with lower $\mu_{i}(t)$ in the past has higher priority being scheduled in order to maintain proportional fairness. Therefore, we formulate the scheduling problem of assigning RBs of CCs and MCSs to UEs as maximizing the weighted sum of transmission rates of UEs at TTI $t$. To simplify notation, the TTI index $t$ is omitted in the following discussion. Let $i, j, k$, and $l$ be UE, $\mathrm{CC}, \mathrm{RB}$, and MCS index respectively, where $i \in M:=$ $\{1, \ldots, m\}, j \in N:=\{1, \ldots, n\}, k \in P:=\{1, \ldots, p\}$, and $l \in$ $Q:=\{1, \ldots, q\}$. The scheduling problem is formulated as solving the following object function:

$$
\max \sum_{i=1}^{m} \sum_{j=1}^{n} \sum_{k=1}^{p} \sum_{l=1}^{q} w_{i} \cdot x_{i, j, k, l} \cdot r_{i, j, k, l}
$$


subjected to the following constraints:

$$
\begin{gathered}
\sum_{i=1}^{m} \sum_{l=1}^{q} x_{i, j, k, l} \leq 1, j \in N, k \in P, \\
\sum_{l=1}^{q} \max _{k \in P} x_{i, j, k, l} \leq 1, i \in M, j \in N, \\
\sum_{l=Q_{i, j, k}+1}^{q} x_{i, j, k, l}=0, i \in M, j \in N, k \in P, \\
\sum_{j=1}^{n} \max _{k \in P} \max _{l \in Q} x_{i, j, k, l} \leq \mathrm{z}, i \in M, \\
x_{i, j, k, l} \in\{0,1\},
\end{gathered}
$$

where $w_{i}$ is a priority weight of $\mathrm{UE} i, x_{i, j, k, l}$ is an indicator variable to denote whether or not RB $k$ of CC $j$ with MCS $l$ is assigned to UE $i$, and $r_{i, j, k, l}$ is the achieved transmission rate for UE $i$ on RB $k$ of CC $j$ with MCS $l$, which is given by the following equation:

$$
r_{i, j, k, l}= \begin{cases}r_{l} & \text { if } l \leq Q_{i, j, k} \\ 0 & \text { otherwise }\end{cases}
$$

If MCS $l$ used by UE $i$ exceeds the allowed rate limitation on $\mathrm{RB} k$ of CC $j$ (i.e., $l>Q_{i, j, k}$ ), it could result in high error rates for RB $k$ of $C C j$ such that $r_{i, j, k, l}$ equals to zero. The constraint in inequality (2) states the restriction that each $\mathrm{RB}$ of any CC is assigned up to one UE. The constraint in (3) ensures that a UE can only use an MCS for its assigned CC. The constraint in (4) dictates that UE $i$ does not select an MCS that UE $i$ cannot use on RB $k$ of CC $j$. The constraint in (5) restricts that a UE can employ at most $z$ CCs. In this paper, we show the hardness in computational complexity of the above scheduling problem and propose a greedy algorithm that maximizes the objective as stated in (1) while satisfying constraints in (2)-(6).

\subsection{Hardness Result}

Lee et al. [20] formulated a radio resource scheduling problem in the LTE systems as a maximization problem. This problem concerns a task of assigning RBs and one of two MIMO modes to each UE at each TTI to maximize the system throughput while maintaining proportional fairness among all UEs. This task subjects to the following constraints: (i) Each RB is assigned up to one UE; (ii) Only one MIMO operational mode can be selected for all assigned RBs for a UE at any TTI. Since LTE systems do not support CA configuration, the authors only consider assigning RBs of one CC.

The authors show that the aforementioned problem is NP-hard. This problem is equivalent to a special case of the radio resource scheduling problem formulated in Section 3.3 under which the system has only one CC and two MCSs for data transmission; the selection of MCSs in our formulated problem can be considered as the equivalent of the selection of MIMO modes in the aforementioned problem. Thus, our formulated problem can be reduced to the aforementioned problem and therefore is NP-hard. In this paper, we shall propose a greedy algorithm to find a suboptimal solution.

\section{Proposed Scheme}

\subsection{Proposed Greedy Algorithm}

Since the scheduling problem is intractable, in this section, we present a greedy algorithm to find a sub-optimal solution. The objective of this algorithm is to maximize the system throughput while maintaining proportional fairness of radio resource allocation among all UEs. To maintain proportional fairness of radio resource allocation among all users, the weighted transmission rate of UE $i$ on RB $k$ of CC $j$ with MCS $l$ is first calculated for all $i, j, k$, and $l$. An assignment of a CC with its associated MCS to a UE is formulated as a gain, which is a function of the weighted transmission rates, and gains of all combinations of UEs, CCs, and MCSs are calculated in each iteration. An assignment with higher gain indicates that the UE can transmit more data by using the MCS with higher rate on the assigned CC and has lower average transmission rate over the accumulated time. Then, iteratively the highest-gain assignment is chosen until all UEs are assigned CCs together with MCSs or no better assignment with higher gain can be found. The greedy algorithm is run for every TTI. The detailed procedure of this algorithm is as follows:

1. Let $U$ be the set of all pair $(i, j)$ of possible assignments of CC $j$ to UE $i$. Initially, $U$ is the set of all combinations of UEs and CCs, i.e., $U=\{(i, j) \mid i \in$ $M, j \in N\}$. Let $V(j, k)$ be the weighted transmission rate of the UE currently being assigned RB $k$ of CC $j$. Since all RBs of all CCs are not assigned to any UE at the initial stage, $V(j, k)$ is set to zero for each $j$ and $k$ initially. Let $v(i, j, k, l)$ be the weighted transmission rate of UE $i$ on RB $k$ of CC $j$ with MCS $l$, which is given by the following equation:

$$
v(i, j, k, l)=w_{i} \cdot r_{i, j, k, l} .
$$

We first update $w_{i}$ and $r_{i, j, k, l}$ and then the weighted transmission rate $v(i, j, k, l)$ is calculated for all $i, j, k$, and $l$.

2. Let $g(i, j, l)$ be the gain of the assignment of CC $j$ with MCS $l$ to UE $i . g(i, j, l)$ is obtained by the following equation:

$$
g(i, j, l)=\sum_{k=1}^{p} \max (0, v(i, j, k, l)-V(j, k)) .
$$

The gains of all possible assignments (i.e., all combinations of pairs in $U$ and MCSs) are calculated in order to find the assignment $\left(i^{*}, j^{*}, l^{*}\right)$ with the largest gain:

$$
\left(i^{*}, j^{*}, l^{*}\right)=\operatorname{argmax}_{(i, j) \in \boldsymbol{U}, l \in Q} g(i, j, l) .
$$

Then, CC $j^{*}$ with MCS $l^{*}$ is assigned to $\operatorname{UE} i^{*}$.

3. For each RB $k$ on $\mathrm{CC} j^{*}$, if the weighted transmission rate of $\mathrm{UE} i^{*}$ on RB $k$ of CC $j^{*}$ is higher than that of 


\begin{tabular}{|c|c|c|}
\hline & RB 1 & RB 2 \\
\hline UE 1 & 3 & 2 \\
\hline UE 2 & 2 & 3 \\
\hline
\end{tabular}

(a) The index of the highest-rate MCS that can be used by each $\mathrm{UE}$ on each RB.

\begin{tabular}{|l|c|c|}
\hline & RB 1 & RB 2 \\
\hline Assigned UE & 1 & 2 \\
\hline Associated MCS & 2 & 3 \\
\hline
\end{tabular}

(c) RB 2 with MCS 3 is reassigned to UE 2 .

Fig. 2. An example of reassigning MCS

the UE currently being assigned RB $k$ of $\mathrm{CC} j^{*}$, i.e., $v\left(i^{*}, j^{*}, k, l^{*}\right)>V\left(j^{*}, k\right), \mathrm{RB} k$ of $\mathrm{CC} j^{*}$ is reassigned to UE $i^{*}$, and $V\left(j^{*}, k\right)$ is set to $v\left(i^{*}, j^{*}, k, l^{*}\right)$. An RB with higher weighted transmission rate indicates that it is utilized more effectively.

4. The pair $\left(i^{*}, j^{*}\right)$ is removed from $\mathbf{U}$ so that the assignment of CC $j^{*}$ to UE $i^{*}$ will not be further considered. Moreover, since a UE can be assigned at most $z$ CCs, if $\mathrm{UE} i^{*}$ has been assigned $z \mathrm{CCs}$, all pairs in $\mathbf{U}$ corresponding to $\mathrm{UE} i^{*}$ are removed so that $\mathrm{UE} i^{*}$ will not be assigned any other CCs.

5. Repeat steps 2-4 until either of the following conditions is satisfied: (i) All UEs have decided which CCs to employ, i.e., all pairs are removed from $U$; (ii) No assignment that can improve the utilization efficiency of any RB of any CC can be found, i.e., $\mathrm{g}\left(i^{*}, j^{*}, l^{*}\right)$ equals to zero.

We can further improve the performance of this algorithm by reassigning the MCS used by each UE $i$ on CC $j^{*}$ in step 3. Since some RBs of $\mathrm{CC} j^{*}$ have already been assigned to UE $i$, and part of these RBs is reassigned to UE $i^{*}$ as in step 3, the remaining RBs may be able to use MCS $l^{\prime}$ with higher rate for transmitting more data. The MCS $l^{\prime}$ that UE $i$ uses on CC $j^{*}$ is obtained by the following equation:

$$
l^{\prime}=\max _{k \in N_{i, j^{*}}} Q_{i, j^{*}, k},
$$

where $N_{i, j^{*}}$ is the set of RBs of CC $j^{*}$ assigned to UE $i$, and $Q_{i, j^{*}, k}$ is the index of the highest-rate MCS that can be used by UE $i$ on RB $k$ of CC $j^{*}$. If MCS $l^{\prime}$ is assigned to UE $i$ for the transmission on CC $j^{*}, V\left(j^{*}, k\right)$ is set to $v\left(i, j^{*}, k, l^{\prime}\right)$ for each RB $k$ of CC $j^{*}$ assigned to UE $i$. An example is shown in Fig. 2, where there are two UEs in the system with one CC of two RBs. Fig. 2a shows the index of the highest-rate MCS that can be used by each UE on each RB. Initially, assume that both RBs with MCS 2 are assigned to UE 1 (Fig. 2b). Since the weighted transmission rate of UE 2 on RB 2 is higher than that of UE 1 on RB 2, and thus RB 2 with MCS 3 is reassigned to UE 2 (Fig. 2c). From Figs. 2a and 2c, UE 1 can use higher-rate MCS 3 to transmit data on RB 1 . Therefore, the scheduler assigns MCS 3 to UE 1 (Fig. 2d).

The pseudo code of the proposed greedy algorithm for the downlink radio resource allocation is shown in Algorithm 1 as follows:

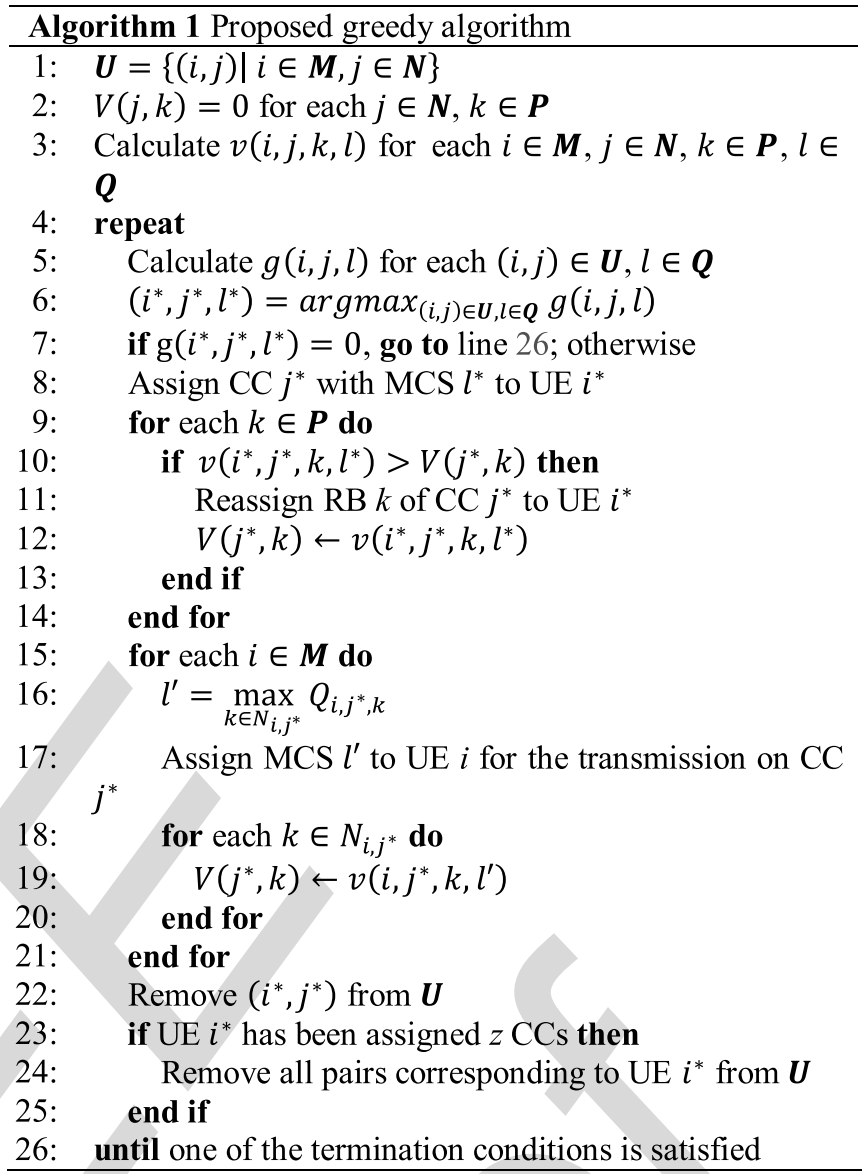

\subsection{Computational Complexity}

As shown in Algorithm 1, line 3 computes all weighted transmission rates, with the computational complexity of $\mathrm{O}(m n p q)$, followed by iterations of the main assignment procedure (lines 4-26). Each iteration computes: (i) All possible gains (line 5), (ii) Reassigning some RBs of CC $j^{*}$ to UE $i^{*}$ (line 9-14) and (iii) Reassigning MCSs to UEs for the transmission on CC $j^{*}$ (lines 15-21), with the complexity of $\mathrm{O}(m n p q), \mathrm{O}(p)$, and $\mathrm{O}(m p)$ respectively. Note that the complexity of the computation of a gain is $\mathrm{O}(p)$. Since the maximum number of iterations is $m \times z$, the total complexity of this algorithm is $\mathrm{O}\left(m^{2} z n p q\right)$.

Computation of Algorithm 1 can be speeded up by the following simplifications: (i) After all possible gains are calculated (line 5), if the largest gain corresponding to UE $i$ equals to zero, the pairs corresponding to UE $i$ can be removed from $\mathbf{U}$; (ii) In the first iteration of the main assignment procedure (lines 4-26), all possible gains are calculated, and only the CC of the largest-gain assignment is assigned to a UE. Therefore, in each iteration except the first one, the algorithm only needs to re-calculate the gains of the assigned CC in the last iteration. The gains of other CCs do not need to be re-calculated since the gains equal to those in the last iteration. Therefore, the total complexity of this algorithm can be reduced to $\mathrm{O}(m n p q+m p q(m z-1))=$ $O(m p q(n+m z-1))$.

\subsection{Performance Analysis}

In this section, we show that Algorithm 1 can guarantee at least half of the performance of the optimal solution. Before 
analyzing the performance of Algorithm 1, two basic definitions about matroid and nondecreasing submodular set function are presented [21].

Definition 1. A system $\mathcal{M}=(\Omega, I)$ is called a matroid, where $\Omega$ is a finite set and $I$ is a nonempty collection of subsets of $\Omega$, satisfying the following properties: (i) If $A \in I$ and $B \subset A$, then $B \in I$; (ii) For all $A \subseteq \Omega$, every maximal member of $I(A)=\{B \mid B \in I, B \subseteq A\}$ has the same cardinality. Note that a set $S$ is called maximal with a property that there is no set $T$ strictly containing $S$.

Definition 2. Given a finite set $\Omega$, a real-valued function $f(\cdot)$ defined on the subsets of $\Omega$ is called a nondecreasing set function, satisfying that for all $A \subset \Omega$ such that $a \in \Omega-A$,

$$
f(A \cup\{a\})-f(A) \geq 0 .
$$

Furthermore, $f(\cdot)$ is called a nondecreasing submodular set function, also satisfying that for all $A, B \subset \Omega$ such that $B \subset A, a \in \Omega-A$,

$$
f(A \cup\{a\})-f(A) \leq f(B \cup\{a\})-f(B) .
$$

The problem of maximizing a nondecreasing submodular set function over a matroid is

$$
\max \{f(A) \mid A \in I\} .
$$

In [22], Fisher et al. has proposed a greedy algorithm (illustrated in Algorithm 2) to address problem (12) and showed that it can guarantee at least half of the performance of the optimal solution.

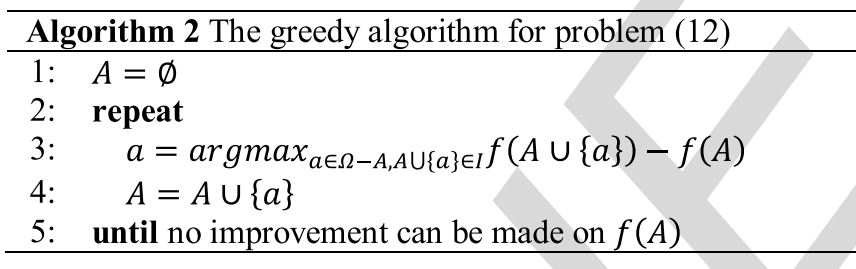

Lemma 1. Algorithm 2 can guarantee at least half of the performance of the optimal solution for problem (12).

We next analyze the performance of Algorithm 1.

Theorem 1. Algorithm 1 can guarantee at least half of the performance of the optimal solution for maximizing the objective as stated in (1) while satisfying constraints in (2)-(6).

Proof. The proof will be divided into the following three steps:

1. We construct a matroid $\mathcal{M}=(\Omega, I)$, where $\Omega=$ $\{(i, j, l) \mid i \in M, j \in N, l \in Q\}$ and $I=\{A \mid A \subset \Omega\}$. We show that it satisfies the matroid property (i): Given $A \in I$ and $B \subset A$, then $B \subset A \subset \Omega \Rightarrow B \in I$. Next, we show that it also satisfies the matroid property (ii): Given $A \subseteq \Omega$ and let $I(A)=$ $\{B \mid B \in I, B \subseteq A\}$, then $I(A) \subset I$. Since $A \subset \Omega$, we have $A \in I$ and therefore $A \in I(A)$. Since $A$ is the largest possible subset contains $A$, we have a unique maximal member of $I(A)$. Thus, the cardinality of all maximal member of $I(A)$ is equal.
2. We map the objective function (1) to a nondecreasing submodular set function $f(\cdot)$ over $\mathcal{M}=(\Omega, I)$, where $f(\cdot)$ on each subset $A \subset \Omega$ is defined as

$$
f(A)=\sum_{j=1}^{n} \sum_{k=1}^{p} \max _{(i, j, l) \in A} w_{i} r_{i, j, k, l} .
$$

We show that $f(\cdot)$ is a nondecreasing set function. For any $A \subset \Omega,\left(i_{0}, j_{0}, l_{0}\right) \in \Omega-A$,

$$
\begin{aligned}
f(A & \left.\cup\left\{\left(i_{0}, j_{0}, l_{0}\right)\right\}\right) \\
& =\sum_{j=1}^{n} \sum_{k=1}^{p} \max _{(i, j, l) \in A \cup\left\{\left(i_{0}, j_{0}, l_{0}\right)\right\}} w_{i} r_{i, j, k, l} \\
& \geq \sum_{j=1}^{n} \sum_{k=1}^{p} \max _{(i, j, l) \in A} w_{i} r_{i, j, k, l}=f(A) .
\end{aligned}
$$

Next, we show that $f(\cdot)$ is a submodular set function.

For any $A, B \subset \Omega, B \subset A,\left(i_{0}, j_{0}, l_{0}\right) \in \Omega-A$,

$$
\begin{aligned}
f\left(A \cup\left\{\left(i_{0}, j_{0}, l_{0}\right)\right\}\right)-f(A) & \\
= & \sum_{j=1}^{n} \sum_{k=1}^{p} \max _{(i, j, l) \in A \cup\left\{\left(i_{0}, j_{0}, l_{0}\right)\right\}} w_{i} r_{i, j, k, l} \\
& -\sum_{j=1}^{n} \sum_{k=1}^{p} \max _{(i, j, l) \in A} w_{i} r_{i, j, k, l} \\
= & \left(\sum_{j=1, j \neq j_{0}}^{n} \sum_{k=1}^{p} \max _{(i, j, l) \in A} w_{i} r_{i, j, k, l}\right. \\
& \left.+\sum_{k=1}^{p} \max _{\left(i, j_{0}, l\right) \in A \cup\left\{\left(i_{0}, j_{0}, l_{0}\right)\right\}} w_{i} r_{i, j_{0}, k, l}\right) \\
& -\left(\sum_{j=1, j \neq j_{0}}^{n} \sum_{k=1}^{p} \max _{(i, j, l) \in A} w_{i} r_{i, j, k, l}+\sum_{k=1}^{p} \max _{\left(i, j_{0}, l\right) \in A} w_{i} r_{i, j_{0}, k, l}\right) \\
= & \sum_{k=1}^{p} \max \left(0, w_{i_{0}} r_{i_{0}, j_{0}, k, l_{0}}-\max _{\left(i, j_{0}, l\right) \in A} w_{i} r_{i, j_{0}, k, l}\right) .
\end{aligned}
$$

Since $A \supset B, \max _{\left(i, j_{0}, l\right) \in A} w_{i} r_{i, j_{0}, k, l} \geq \max _{\left(i, j_{0}, l\right) \in B} w_{i} r_{i, j_{0}, k, l}$, $f\left(A \cup\left\{\left(i_{0}, j_{0}, l_{0}\right)\right\}\right)-f(A)$

$\leq \sum_{k=1}^{p} \max \left(0, w_{i_{0}} r_{i_{0}, j_{0}, k, l_{0}}-\max _{\left(i, j_{0}, l\right) \in B} w_{i} r_{i, j_{0}, k, l}\right)$

$=\sum_{j=1}^{n} \sum_{k=1}^{p} \max _{(i, j, l) \in B \cup\left\{\left(i_{0}, j_{0}, l_{0}\right)\right\}} w_{i} r_{i, j, k, l}$

$-\sum_{j=1}^{n} \sum_{k=1}^{p} \max _{(i, j, l) \in B} w_{i} r_{i, j, k, l}$

$=f\left(B \cup\left\{\left(i_{0}, j_{0}, l_{0}\right)\right\}\right)-f(B)$.

3. We show that the goal of Algorithm 1 can be mapped to the goal of Algorithm 2. Let $A \subset \Omega$ be the set of combinations of $(i, j, l)$, where $i \in M, j \in N, l \in Q$ and CC $j$ with MCS $l$ is assigned to UE $i$ in previous iterations in Algorithm 1. The goal of Algorithm 1 (line 6) is that the optimal assignment $\left(i^{*}, j^{*}, l^{*}\right)$ is selected to maximize $g\left(i^{*}, j^{*}, l^{*}\right)$ in each iteration. The goal 
TABLE 2

Simulation Settings

\begin{tabular}{|c|c|}
\hline Parameter & Setting \\
\hline Inter-site distance (ISD) & $500 \mathrm{~m}$ \\
\hline Component carriers & $\begin{array}{l}4 \times 5 \mathrm{MHz} \text { DL CCs are at } \\
S 1\{2 \mathrm{GHz}, 2 \mathrm{GHz}, 2 \mathrm{GHz}, 2 \mathrm{GHz}\} \& \\
S 2\{800 \mathrm{MHz}, 800 \mathrm{MHz}, 2 \mathrm{GHz}, 2 \mathrm{GHz}\}\end{array}$ \\
\hline Number of RBs per CC & 25 (12 subcarriers per $\mathrm{RB})$ \\
\hline Path loss & $\begin{array}{l}\mathrm{L}=\mathrm{I}+37.6 \log _{10}(R), \mathrm{R} \text { in } \mathrm{km} \\
\mathrm{I}=128.1(2 \mathrm{GHz}) \\
\mathrm{I}=120.9(900 \mathrm{MHz})[23]\end{array}$ \\
\hline Penetration loss & $20 \mathrm{~dB}$ \\
\hline Shadowing loss & $\begin{array}{l}\text { Gaussian distribution with zero mean } \\
\text { and standard deviation } 8 \mathrm{~dB}\end{array}$ \\
\hline Multipath & Jakes' model [24] \\
\hline Available MCSs & $\begin{array}{l}29 \text { possible MCSs are available as de- } \\
\text { fined in 3GPP TS } 36.213 \text { [17] }\end{array}$ \\
\hline Number of UEs per cell & $10,20,30,40,50$ \\
\hline UE speed & $1-15 \mathrm{mps}$ \\
\hline Traffic model & $\begin{array}{l}\text { Backlogged traffic model (fixed number of } \\
\text { UEs with full buffer) }\end{array}$ \\
\hline TTI (Subframe) & $1 \mathrm{~ms}$ \\
\hline Granularity of CSI feedback & 100 TTIs \\
\hline Granularity of scheduling & $1 \mathrm{TTI}$ \\
\hline
\end{tabular}

of Algorithm 2 (line 3) is that the optimal element $a=\left(i^{*}, j^{*}, l^{*}\right)$ is selected to maximize $f\left(A \cup\left\{\left(i^{*}, j^{*}, l^{*}\right)\right\}\right)-f(A)$ in each iteration. If $g\left(i^{*}, j^{*}, l^{*}\right)$ is equivalent to $f\left(A \cup\left\{\left(i^{*}, j^{*}, l^{*}\right)\right\}\right)-$ $f(A)$, the goals of both algorithms are equivalent. Therefore, we show that $g\left(i^{*}, j^{*}, l^{*}\right)=f\left(A \cup\left\{\left(i^{*}\right.\right.\right.$, $\left.\left.\left.j^{*}, l^{*}\right)\right\}\right)-f(A)$ by

$$
g\left(i^{*}, j^{*}, l^{*}\right)=\sum_{k=1}^{p} \max \left(0, v\left(i^{*}, j^{*}, k, l^{*}\right)-V\left(j^{*}, k\right)\right) .
$$

$$
\text { Since } \begin{aligned}
v\left(i^{*}, j^{*}, k, l^{*}\right) & =w_{i *} r_{i^{*}, j^{*}, k, l^{*}} \text { and } V\left(j^{*}, k\right) \\
& =\max _{\left(i, j^{*}, l\right) \in A} w_{i} r_{i, j^{*}, k, l},
\end{aligned}
$$

$$
\begin{aligned}
& g\left(i^{*}, j^{*}, l^{*}\right) \\
& \quad=\sum_{k=1}^{p} \max \left(0, w_{i^{*}} r_{i^{*}, j^{*}, k, l^{*}}-\max _{\left(i, j^{*}, l\right) \in A} w_{i} r_{i, j^{*}, k, l}\right) \\
& \quad=f\left(A \cup\left\{\left(i^{*}, j^{*}, l^{*}\right)\right\}\right)-f(A) .
\end{aligned}
$$

The goals of both algorithms are equivalent so that they have the same performance bound. Thus, by Lemma 1, Algorithm 1 can guarantee at least half of the performance of the optimal solution for maximizing the objective as stated in (1) while satisfying constraints in (2)-(6).

\section{Simulation}

\subsection{Simulation Settings}

In order to evaluate the performance of our proposed scheme, we modified LTE-EPC network simulator [25] that is based on ns-3 network simulator [26] to conduct a series of LTE-A downlink system-level simulations. Table 2 summarizes a list of main parameters used in the simulations. We evaluate performance of two possible CA scenarios S1

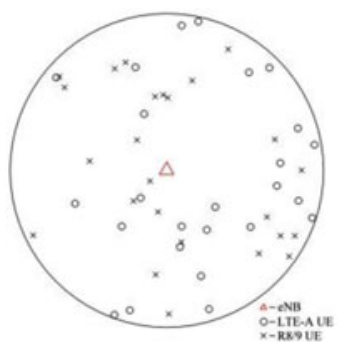

(a) Random deployment.

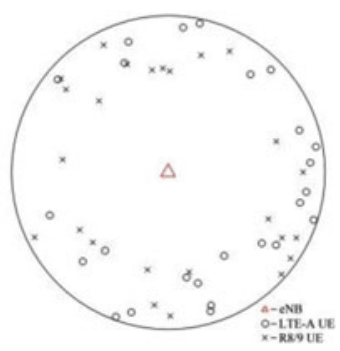

(b) Cell-edge deployment.

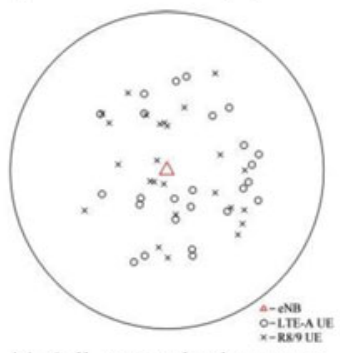

(c) Cell-center deployment.

Fig. 3. UE deployment scenarios.

and S2 with four downlink (DL) CCs of $5 \mathrm{MHz}$ bandwidth. In 51 , the four CCs are at $2 \mathrm{GHz}$ frequency band, whereas, in $S 2$, they are at $800 \mathrm{MHz}, 800 \mathrm{MHz}, 2 \mathrm{GHz}$, and $2 \mathrm{GHz}$ frequency band, respectively. The number of UEs per cell varies from 10 to 50. Both LTE Release 8/9 and LTE-A UEs are supported in the system. Furthermore, LTE Release 8/9 UEs can employ one CC, while LTE-A UEs can employ two CCs simultaneously. The performance under various ratios of LTE-A UEs is considered. The performance is evaluated under three UE deployment scenarios, namely Random, Cell-edge, and Cell-center, as shown in Fig. 3. In Random (Fig. 3a), UEs are scattered and equally-distributed over the whole area of the cell, whereas in Cell-edge (Fig. 3b) and Cell-center (Fig. 3c), UEs are distributed near the cell-edge and cell-center, respectively. The UEs are mobile with various velocities from 1 to $15 \mathrm{mps}$. The simulation process is conducted with 100 simulation runs, and the duration of each simulation run is 10 seconds (i.e., 10,000 TTIs).

Due to ease of accessibility, we compare the performance of our scheme with the scheme proposed in [8]. The scheme in [8] includes CC assignment and packet scheduling functions. CC assignment can be performed by Least Load approach [27] or random carrier approach [27]. The goal of least load is that each UE is assigned the CCs that have the least number of UEs, while the goal of random carrier is that each UE is assigned CCs randomly. After CCs are assigned, the scheme in [8] assigns the RBs of each CC to UEs by its packet scheduling function. However, its packet scheduling function does not observe the MCS constraint. Therefore, we adopt the packet scheduling function proposed in [15] since it considers the MCS constraint in LTE systems. We further modify it to support CA in LTE-A systems and name it SS. After CC assignment is performed, SS is executed in the following steps:

1. The scheduler selects a CC. Each UE on this CC chooses the highest-rate MCS based on the channel conditions of all RBs and then calculates its weighted transmission rate. 


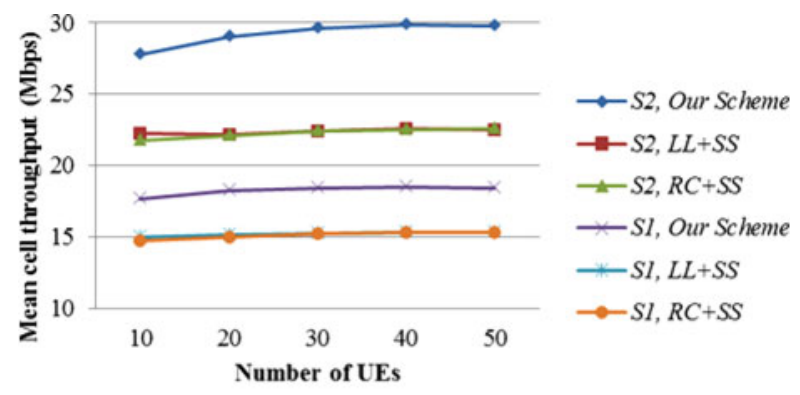

(a) Random deployment.

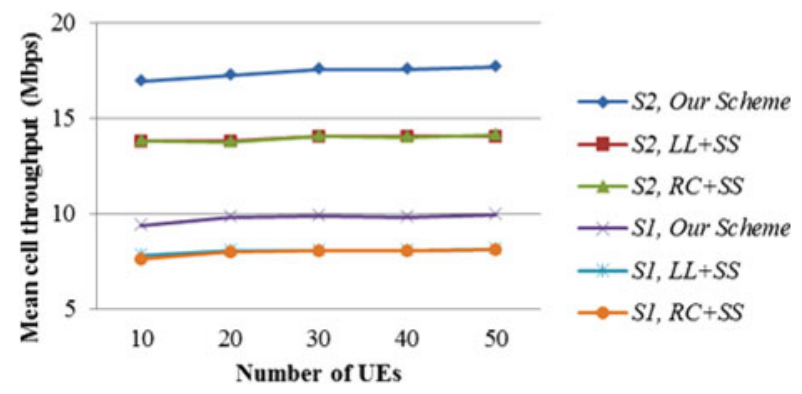

(b) Cell-edge deployment.

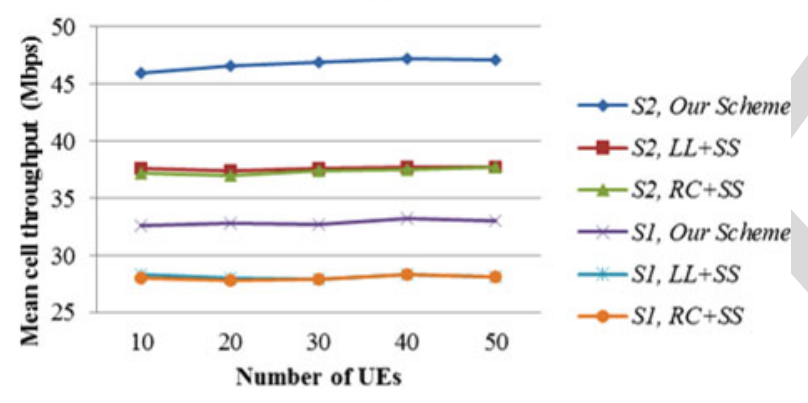

(c) Cell-center deployment.

Fig. 4. Mean cell throughput versus number of UEs.

2. The UE with the highest weighted transmission rate is assigned the RBs which can support its selected MCS.

3. The UE with next highest weighted transmission rate is assigned some of the remaining RBs.

4. Repeat step 3 until all RBs of this selected CC have been assigned.

5. Repeat steps 1-4 until all CCs have been selected.

In conclusion, the CC assignment is performed by least load or random carrier approach, while the packet scheduling is executed by SS. Thus, we compare our scheme to least load with SS $(L L+S S)$ and random carrier with SS $(R C+S S)$ schemes.

\subsection{Simulation Results}

We measure the mean cell throughput, which is the mean aggregated throughput from all UEs in the cell. The mean cell throughput for different schemes with various numbers of UEs (50 percent of UEs are LTE-A) under different scenarios is shown in Fig. 4. It is expected that the Cell-center deployment has the highest mean cell throughput while the Cell-edge deployment has the lowest mean cell throughput for all schemes. In all scenarios, our scheme outperforms all
TABLE 3

Throughput Improvement

\begin{tabular}{|c|c|c|c|}
\hline Deployment & Scenario & Min. at \# of UEs & Max. at \# of UEs \\
\hline \multirow{2}{*}{ Random } & $\mathrm{S} 1$ & $18.9 \% @ 10$ & $20.7 \% @ 20$ \\
\cline { 2 - 4 } & $\mathrm{S} 2$ & $24.6 \% @ 10$ & $32.3 \% @ 50$ \\
\hline \multirow{2}{*}{ Cell-edge } & $\mathrm{S} 1$ & $21.3 \% @ 10$ & $22.4 \% @ 20$ \\
\cline { 2 - 4 } & $\mathrm{S} 2$ & $22.7 \% @ 10$ & $25.7 \% @ 50$ \\
\hline \multirow{2}{*}{ Cell-center } & $\mathrm{S} 1$ & $15.7 \% @ 10$ & $17.57 @ 20$ \\
\cline { 2 - 4 } & $\mathrm{S} 2$ & $22.7 \% @ 10$ & $25.33 \% @ 50$ \\
\hline
\end{tabular}

the other schemes due to the following reasons: (i) Our scheme assigns CCs to UEs by considering the channel quality of CCs, and it can reassign the CCs with higher transmission rate to UEs at each TTI. However, the other schemes assign CCs to UEs by considering the traffic load of CCs or using randomized method, and they cannot change the assigned CCs in each UE. (ii) Our scheme can reassign the CC with a higher-rate MCS to other UEs after this CC is assigned to a UE, while the other schemes only assign MCSs of CCs to UEs at the initial stage. From Fig. 4, we note that the mean cell throughput of scheme $L L+S S$ are not much different from that of scheme $R C+S S$. Therefore we show in Table 3 the throughput improvement of our proposed scheme comparing to the average throughput of $L L+S S$ and $R C+S S$ in each scenario and deployment. Our scheme improves the mean cell throughput with 15.732.3 percent, comparing to the other schemes.

The mean cell throughput is influenced by the following factors: (i) When a cell is sparse with UEs, the more are UEs in the cell, the higher is the number of UEs that are assigned RBs with higher channel quality, leading to an increase in the mean cell throughput; (ii) When a cell becomes denser with UEs, the fewer are RBs with higher channel quality that can be assigned to each UE, resulting in lower mean cell throughput. Since the mean cell throughput interacts with the aforementioned factors, as shown in Fig. 4, it first increases and then changes slightly as the number of UEs sharing the system capacity increases. We note in Table 3 that the maximum throughput improvement for scenario $S 1$ occurs when the number of UEs equals to around 20 while that of scenario S2 occurs around 50. This is due to the fact that in $S 2$ the CCs with higher frequency $(2 \mathrm{GHz})$ suffer from larger path loss than those with lower frequency $(800 \mathrm{MHz})$, there are more UEs being assigned RBs of CCs (800 MHz) with better channel quality in $S 2$ than in $S 1$ as the number of UEs increases. Due to the same above reason, the performance in $S 2$ is generally higher than that in $S 1$.

The results in Figs. 5 and 6 show the mean cell throughput for different schemes with a constant number of UEs but different ratios of LTE-A UEs under different scenarios. In all scenarios, our scheme outperforms all the other schemes due to the aforementioned reasons. Moreover, LTE Release 8/9 UEs can employ only one CC, while LTE-A UEs can employ two CCs simultaneously. Compared with LTE Release 8/9 UEs, LTE-A UEs have higher probability to be allocated the higher-rate RBs of 


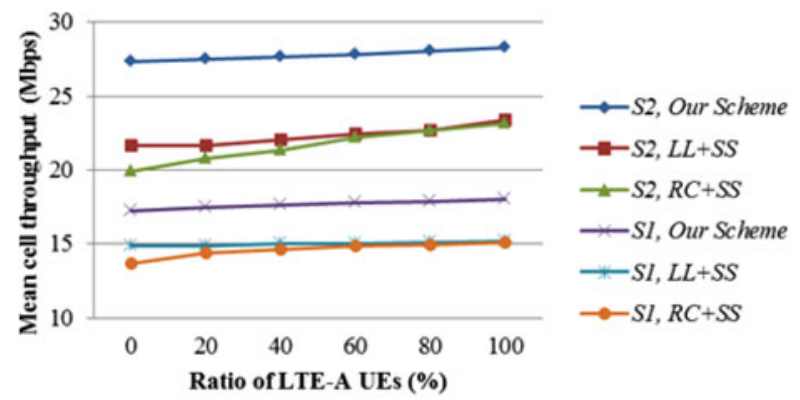

(a) 10 UEs in the cell and Random deployment.

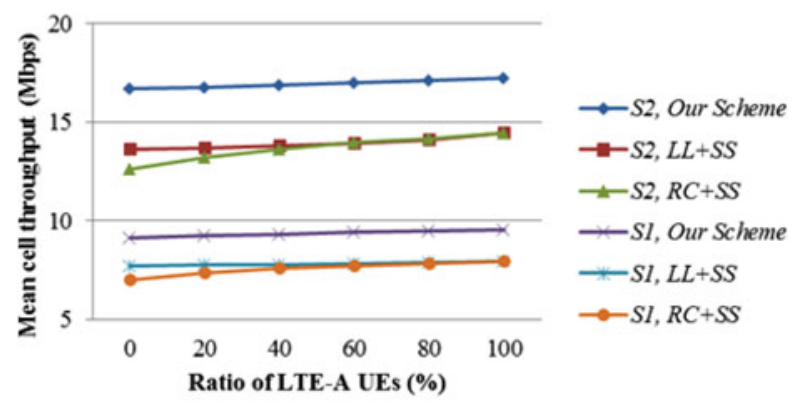

(b) 10 UEs in the cell and Cell-edge deployment.

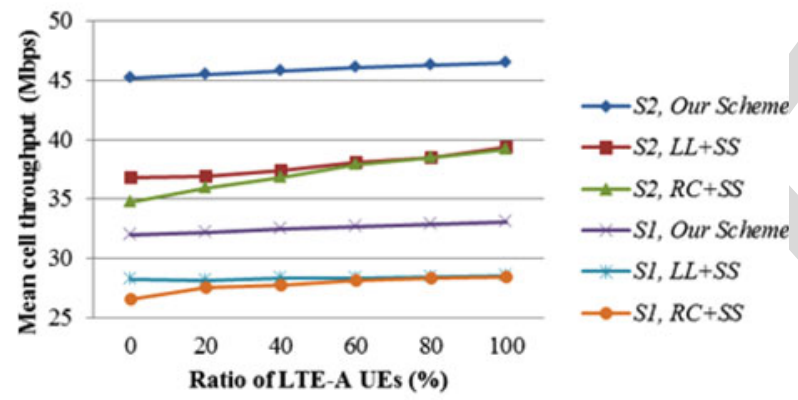

(c) 10 UEs in the cell and Cell-center deployment.

Fig. 5. Mean cell throughput versus ratio of LTE-A UEs (10 UEs).

the assigned CC. Therefore, the mean cell throughput of all schemes increases as the ratio of LTE-A UEs increases when there are 10 UEs in a cell shown in Fig. 5. However, when there are too many UEs sharing the system capacity, each UE of both LTE Release 8/9 and LTE-A can only use fewer RBs to transmit data. Thus, the mean cell throughput of all schemes changes slightly as the ratio of LTE-A UEs increases as the case of 50 UEs shown in Fig. 6. Concluding from the above simulation results, our scheme significantly outperforms other schemes in all scenarios, various deployments and combinations of LTE-A and LTE Release 8/9 UEs, since our scheme can assign CCs to UEs taking into account of the channel quality of CCs, and also it can dynamically reassign the CCs with higher transmission rate to UEs at each TTI.

In order to analyze the degree of fairness among all UEs, we adopt Jain's fairness index [28], denoted as $F$, which is defined by the following equation:

$$
F=\frac{\left(\sum_{i=1}^{m} \mu_{i}\right)^{2}}{m \sum_{i=1}^{m} \mu_{i}^{2}},
$$

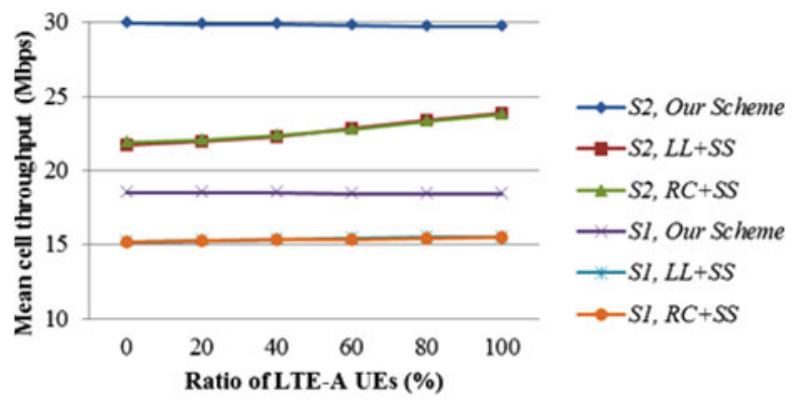

(a) 50 UEs in the cell and Random deployment.

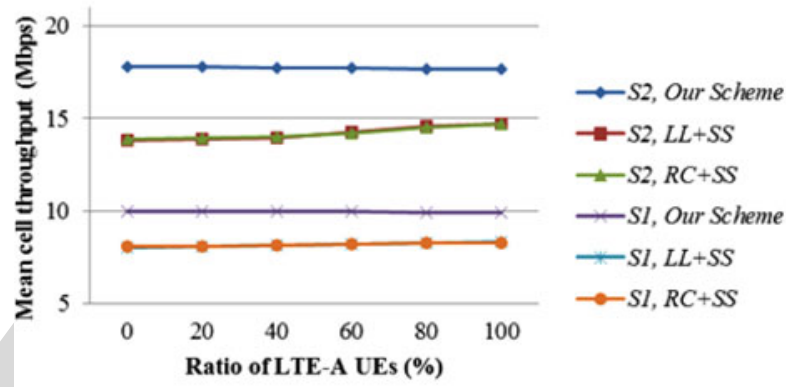

(b) 50 UEs in the cell and Cell-edge deployment.

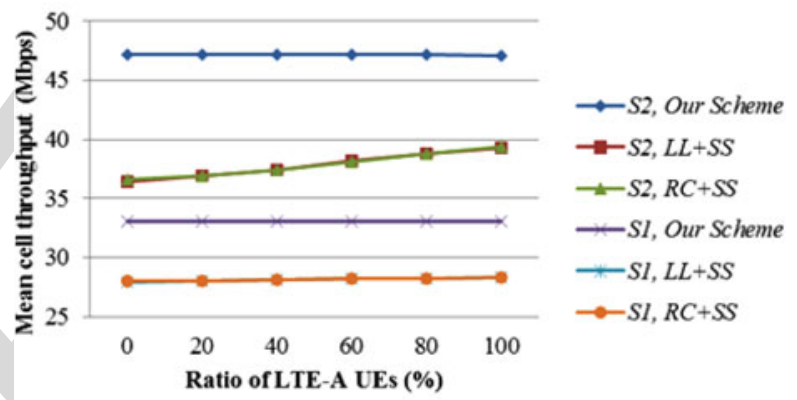

(c) 50 UEs in the cell and Cell-center deployment.

Fig. 6. Mean cell throughput versus ratio of LTE-A UEs (50 UEs).

where $\mu_{i}$ is the average transmission rate of UE $i$. The value of $F$ ranges from $1 / m$ to 1 , and $F=1$ indicates that all UEs have equal average transmission rate. Fig. 7 displays the fairness index for different schemes with various numbers of UEs (50 percent of UEs are LTE-A) under different scenarios. All schemes have approximately equal fairness index in S1. As mentioned earlier, in $S 2$ scenario, $800 \mathrm{MHz}$ $\mathrm{CC}$ has better channel quality than $2 \mathrm{GHz} \mathrm{CC}$. With our scheme in S2, some UEs are more likely to be assigned RBs of CCs $(800 \mathrm{MHz})$ with better channel quality, resulting in lower fairness performance, comparing to other schemes that do not take into account of the channel quality of CCs being assigned, as shown in Fig. 7 .

In the Random and Cell-edge deployments, with our scheme in S2 scenario, those UEs near the cell edge are more likely to be assigned RBs of CCs $(800 \mathrm{MHz})$ with better channel quality, comparing to other schemes, leading to lower fairness indices as shown in Figs. $7 \mathrm{a}$ and $7 \mathrm{~b}$, respectively. As the number of UEs increases, it is expected that the fairness indices decreases as more UEs being assigned RBs of CCs $(800 \mathrm{MHz})$ with better channel quality while other UEs (mostly LTE Release 8/9 UEs) being assigned 


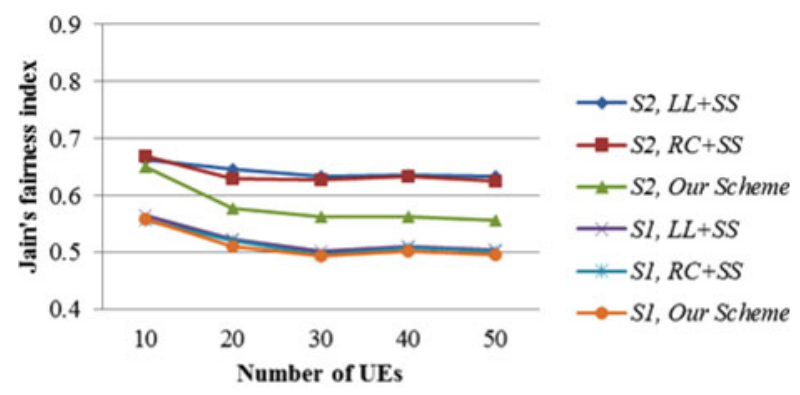

(a) Random deployment.

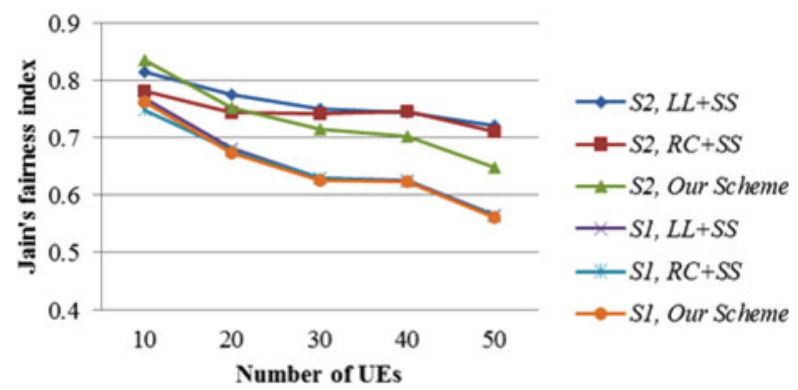

(b) Cell-edge deployment.

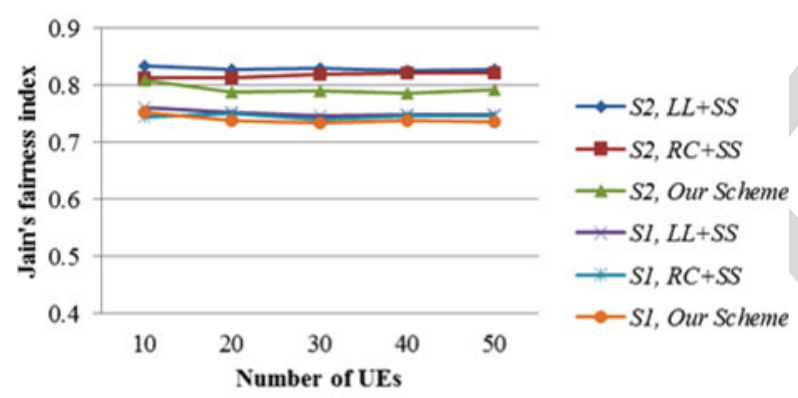

(c) Cell-center deployment.

Fig. 7. Jain's fairness index versus number of UEs.

RBs of CCs (2 GHz) with lower channel quality. In the Cellcenter deployment, all UEs are close to the eNB and so they have almost the equal opportunity to be assigned RBs of CCs with good channel quality. Thus the fairness index of our scheme is only slightly lower than those of other schemes as shown in Fig. 7c.

From the above simulations, we conclude that our scheme has resulted in better throughput performance and lower fairness index than other schemes. However our scheme adopts the proportional fair scheduling, which gives higher priority of assigning RBs to UEs with lower average transmission rate in the past. Therefore, UEs are not likely to suffer starvation although our scheme has lower fairness index.

Finally in this section, in order to compare the performance of our scheme with the optimal solution, we have implemented an optimal scheduler by brute-force search and conducted a series of simulations under a small-scale scenario since the optimal scheduler has intractable computational complexity. In this scenario, the system has two DL $\mathrm{CCs}$ of $1.4 \mathrm{MHz}$ at 2-GHz frequency band. We measure the mean aggregated weighted transmission rate from all UEs in the cell. The mean aggregated weighted transmission

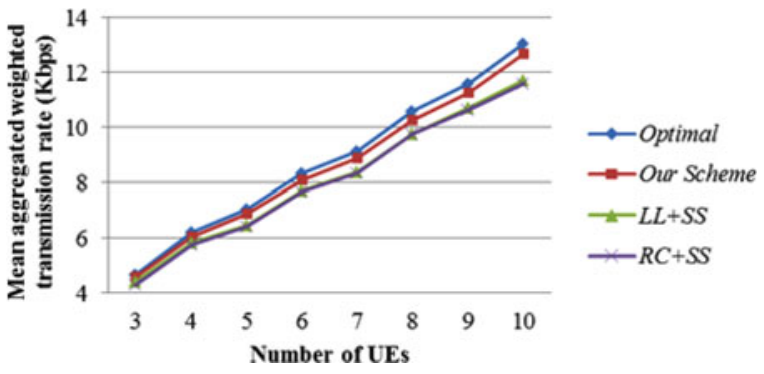

Fig. 8. Mean aggregated weighted transmission rate versus number of UEs under the small-scale scenario.

rate for different schemes with various numbers of UEs (50 percent of UEs are LTE-A) is shown in Fig. 8. Note that our solution is quite close to the optimal solution.

\section{Conclusions}

In this paper, we have investigated the downlink radio resource scheduling problem for LTE-A systems with CA configuration. This problem concerns the allocation of RBs of CCs and MCSs under the MCS constraint as specified in LTE-A standards. We have formulated the problem and proved that it is NP-hard. We therefore developed a novel greedy-based scheme to address this problem. This scheme can effectively maximize the system throughput while maintaining proportional fairness among all UEs. With this scheme, a sub-optimal solution that can guarantee at least half of the performance of the optimal solution can be found. Based on 3GPP specifications, we have conducted a series of LTE-A downlink system-level simulations. The simulation results reveal that the proposed scheme can improve substantially throughput performance compared with the schemes in previous studies. We also analyze fairness of the proposed scheme and show that UEs are not likely to suffer starvation although our scheme has lower fairness index. For future work, we plan to further tackle the radio resource scheduling problem regarding $\mathrm{CA}$ in conjunction with MIMO technique since MIMO technique, which regards the context-dependent selection of operational modes for data transmission, may play an important role in future LTE-A Networks.

\section{ACKNOWLEDGMENTS}

This work is partially supported by Grants 101-2221-E-001024- and 100-2221-E-001-025-MY3 of the National Science Council, Taiwan and Grants of Academia Sinica.

\section{REFERENCES}

[1] "Requirements for Further Advancements for Evolved Universal Terrestrial Radio Access (E-UTRA) (LTE-Advanced))," Technical Report 36.913, 3rd Generation Partnership Project (3GPP), Mar. 2011.

[2] "Evolved Universal Terrestrial Radio Access (E-UTRA) and Evolved Universal Terrestrial Radio Access Network (E-UTRAN); Overall description; Stage 2," TS 36.300, 3rd Generation Partnership Project (3GPP), June 2012.

[3] Z. Shen, A. Papasakellariou, J. Montojo, D. Gerstenberger, and F. $\mathrm{Xu}$, "Overview of 3GPP LTE-Advanced Carrier Aggregation for 4G Wireless Communications," IEEE Comm. Magazine, vol. 50, no. 2, pp. 122-130, Feb. 2012. 
[4] H. Tian, S. Gao, J. Zhu, and L. Chen, "Improved Component Carrier Selection Method for Non-Continuous Carrier Aggregation in LTE Advanced Systems," Proc. IEEE Vehicular Technology Conf., pp. 1-5, Sept. 2011

[5] H. Wang, C. Rosa, and K. Pedersen, "Performance Analysis of Downlink Inter-band Carrier Aggregation in LTE-Advanced," Proc. IEEE Vehicular Technology Conf. (VTC Fall), pp. 1-5, Sept. 2011.

[6] L. Liu, M. Li, J. Zhou, X. She, L. Chen, Y. Sagae, and M. Iwamura, "Component Carrier Management for Carrier Aggregation in LTE Advanced System," Proc. IEEE Vehicular Technology Conf. (VTC Spring), pp. 1-6, May 2011.

[7] A. Jalali, R. Padovani, and R. Pankaj, "Data Throughput of CDMA-HDR a High Efficiency-High Data Rate Personal Communication Wireless System," Proc. IEEE Vehicular Technology Conf. (VTC Spring), vol. 3, pp. 1854-1858, 2000.

[8] Y. Wang, K.I. Pedersen, T.B. Sorensen, and P.E. Mogensen, "Carrier Load Balancing and Packet Scheduling for Multi-Carrier Systems," IEEE Trans. Wireless Comm., vol. 9, no. 5, pp. 1780-1789, May 2010.

[9] Y. Wang, K.I. Pedersen, T.B. Sorensen, and P.E. Mogensen, "Utility Maximization in LTE-Advanced Systems with Carrier Aggregation," Proc. IEEE Vehicular Technology Conf. (VTC Spring), pp. 1-5, May 2011.

[10] "Feasibility Study for Further Advancements for E-UTRA (LTEAdvanced)," TR 36.912, 3rd Generation Partnership Project (3GPP), Mar. 2011.

[11] R. Kwan, C. Leung, and J. Zhang, "Resource Allocation in an LTE Cellular Communication System," Proc. IEEE Seventh Int'l Conf. Comm., pp. 1-5, June 2009.

[12] N. Guan, Y. Zhou, L. Tian, G. Sun, and J. Shi, "QoS Guaranteed Resource Block Allocation Algorithm for LTE Systems," Proc. IEEE Seventh Int'l Conf. Wireless and Mobile Computing, Networking and Comm. (WiMob), pp. 307-312, Oct. 2011

[13] M.E. Aydin, R. Kwan, J. Wu, and J. Zhang, "Multiuser Scheduling on the LTE Downlink with Simulated Annealing," Proc. IEEE Vehicular Technology Conf. (VTC Spring), pp. 1-5, May 2011

[14] M.E. Aydin, R. Kwan, and J. Wu, "Multiuser Scheduling on the LTE Downlink with Meta-Heuristic Approaches," Physical Comm., vol. 9, pp. 257-265, 2012.

[15] R. Kwan, C. Leung, and J. Zhang, "Proportional Fair Multiuser Scheduling in LTE," IEEE Signal Processing Letters, vol. 16, no. 6, pp. 461-464, June 2009.

[16] "Evolved Universal Terrestrial Radio Access (E-UTRA); Physical Channels and Modulation," TS 36.211 3rd Generation Partnership Project (3GPP), June 2012.

[17] "Evolved Universal Terrestrial Radio Access (E-UTRA); Physical Layer Procedures," TS 36.213, 3rd Generation Partnership Project (3GPP), June 2012.

[18] M. Andrews, "A Survey of Scheduling Theory in Wireless Data Networks," IMA Volumes in Mathematics and Its Applications, vol. 143, pp. 1-18, 2007.

[19] H.J. Kushner and P.A. Whiting, "Asymptotic Properties of Proportional-Fair Sharing Algorithms," Proc. IEEE 10th Ann. Allerton Conf. Comm., Control, and Computing, pp. 1051-1059, 2002.

[20] S.-B. Lee, S. Choudhury, A. Khoshnevis, S. Xu, and S. Lu, "Downlink MIMO with Frequency-Domain Packet Scheduling for 3GPP LTE," Proc. IEEE INFOCOM, pp. 1269-1277, Apr. 2009.

[21] G.L. Nemhauser, L.A. Wolsey, and M.L. Fisher, "An Analysis of Approximations for Maximizing Submodular set Functions - I," Math. Programming, vol. 14, no. 1, pp. 265-294, Dec. 1978.

[22] M.L. Fisher, G.L. Nemhauser, and L.A. Wolsey, "An Analysis of Approximations for Maximizing Submodular Set Functions - II," Math. Programming Study, no. 8, pp. 73-87, 1978.

[23] "Physical Layer Aspects for Evolved Universal Terrestrial Radio Access (UTRA)," Technical Report 25.814, 3rd Generation Partnership Project (3GPP), Sept. 2006.

[24] W.C. Jakes, Microwave Mobile Communications. Wiley, Feb. 1975.

[25] "LTE-EPC Network Simulator (LENA)-Iptechwiki," http:// iptechwiki.cttc.es/, 2014.

[26] "ns-3 Network Simulator," http://www.nsnam.org/, 2014.

[27] T. Dean and P. Fleming, "Trunking Efficiency in Multi-Carrier CDMA Systems," Proc. IEEE Vehicular Technology Conf. (VTC Fall), vol. 1, pp. 156-160, 2002.

[28] R. Jain, D.M. Chiu, and W. Hawe, "A Quantitative Measure of Fairness and Discrimination for Resource Allocation in Shared Computer System," Technical Report 301, DEC, Sept. 1984.

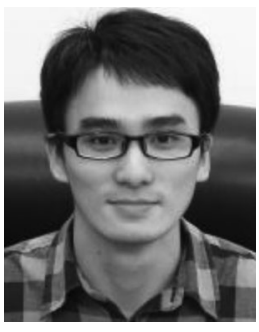

Hong-Sheng Liao received the BE degree from the Department of Computer Science and Information Engineering from the National Dong Hwa University, Taiwan, in 2010, and the MS degree from the Department of Computer Science from National Tsing Hua University, Taiwan, in 2012. $\mathrm{He}$ is currently working at Chunghwa Telecom Co. Ltd, responsible for the development of a content distribution system. His research interests include wireless and mobile network systems.

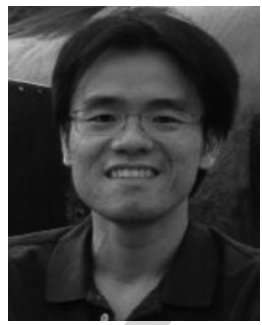

Po-Yu Chen received the BS degree from the Department of Electrical Engineering from Chang Gung University, Taiwan, in 2001, and the MS and PhD degrees from the Institute of Communications Engineering both from National Tsing Hua University, Taiwan, in 2003 and 2009, respectively. He had been a postdoctoral research fellow in the Department of Computer Science, National Tsing Hua University from 2010 to 2012 . He is now a postdoctoral research fellow in the institute of information science of Academia Sinica. His research interests include wireless ad hoc and sensor networks, vehicular ad hoc networks, IoT, and LTE-A networks. $\mathrm{He}$ is a member of the IEEE.

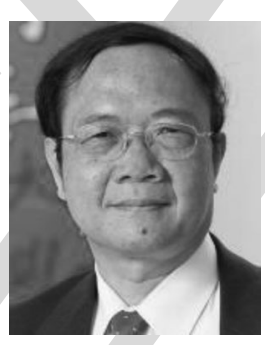

Wen-Tsuen Chen received the BS degree in nuclear engineering from National Tsing Hua University, Taiwan, and the MS and $\mathrm{PhD}$ degrees in electrical engineering and computer sciences both from the University of California, Berkeley, in 1970, 1973, and 1976, respectively. He has been with the National Tsing Hua University since 1976 and is a distinguished chair professor of the Department of Computer Science. He has served as the chairman of the Department, dean of College of Electrical Engineering and Computer Science, and the president of National Tsing Hua University. In March 2012, he joined the Academia Sinica, Taiwan, as a distinguished research fellow of the Institute of Information Science. His research interests include computer networks, wireless sensor networks, mobile computing, and parallel computing. He received numerous awards for his academic accomplishments in computer networking and parallel processing, including Outstanding Research Award of the National Science Council, Academic Award in Engineering from the Ministry of Education, Technical Achievement Award and Taylor L. Booth Education Award of the IEEE Computer Society, and is currently a lifelong National Chair of the Ministry of Education, Taiwan. He is the founding general chair of the IEEE International Conference on Parallel and Distributed Systems and the general chair of the 2000 IEEE International Conference on Distributed Computing Systems among others. He is an IEEE fellow and fellow of the Chinese Technology Management Association.

$\triangleright$ For more information on this or any other computing topic, please visit our Digital Library at www.computer.org/publications/dlib. 